\title{
¿POR QUÉ LOS CURRÍCULA NO PUEDEN EDUCAR? UNA MIRADA RADICAL INCIUSIVA
}

\section{WHY CAN'T CURRICULA EDUCATE? AN INCLUSIVE RADICAL LOOK}

\author{
Dr. Agustín de la Herrán Gascón ${ }^{1}$ \\ Universidad Autónoma de Madrid
}

\section{RESUMEN}

Dentro de la Pedagogía radical e inclusiva se aplica su enfoque radical e inclusivo a la educación, los sistemas educativos y los currículos globalizados, junto a otros aspectos relevantes que les afectan. Como consecuencia de ello, se cuestiona y se explica por qué los currícula están mal planteados de raíz y no sirven para educar plenamente. Se aportan líneas para su reparación y evolución, tanto para la Pedagogía como para la educación y para los propios currículos. A lo largo del texto, se alude y conceptúan originalmente tres nuevas clases

$1 \quad$ - Dr. Agustín de la Herrán Gascón. Doctor en Educación. Investigador Universidad Autónoma de Madrid -UAM, Madrid, España. https://orcid.org/0000-0001-91566971, agustin.delaherran@uam.es de currículo, aunque se hayan presentado en trabajos anteriores: currículo demandado o bidimensional, currículo descarado y currículo radical.

PALABRAS CLAVE: Pedagogía radical e inclusiva, currículo, educación, conciencia

\section{ABSTRACT}

Within radical and inclusive Pedagogy, its radical and inclusive approach is applied to education, education systems and globalized curricula, along with other relevant aspects that affect them. As a result, it questions and explains why curricula are poorly designed at the root and do not serve to fully educate. It provides guidelines for their repair and evolution, both for pedagogy 
and education and for the curricula themselves. Throughout the text, three new kinds of curricula are originally alluded to and conceptualized, even though they have been presented in previous works: demanded or two-dimensional curriculum, shameless curriculum, and radical curriculum.

KEYWORDS: Radical and inclusive Pedagogy, curriculum, education, awareness

\section{INTRODUCCIÓN}

La Pedagogía radical e inclusiva estudia la educación, la enseñanza y la formación desde una metáfora: la educación es como un árbol. Casi siempre muestra la misma parte, que se toma como el todo y, como tal, se conceptúa e investiga. Por esta miopía, nuestra educación se percibe superficialmente y las acciones educativas, aunque puedan ser necesarias, son limitadas. Se dejan de lado cuestiones esenciales, sin cuya inclusión y conciencia curricular, la educación plena no es posible.

En este escrito, el enfoque radical e inclusivo se aplica al paradigma educativo dominante, con énfasis en los sistemas educativos y currícula globalizados del primer mundo. Como consecuencia de ello, se cuestiona y se explica por qué los currícula están mal planteados de raíz y no sirven para educar. Finalmente, se aportan líneas para su reparación y desarrollo.

\section{ALGUNOS PROBLEMAS RADICALES DE LOS CURRÍCULA}

Los currícula son intenciones educativas formuladas socialmente para desarrollarse mediante la educación, frutos de su población y de su época. Dependen de las culturas y de la noción de educación acorde. Por eso son productos relativos. Los ha habido al menos desde la Edad Antigua (Herrán, 2012).
Nuestra época globalizada es ostensible por sus logros, pero sobre todo por sus desaciertos. Nuestra educación no está siendo útil para corregirlos, por no comprenderse cabalmente y estar mal fundada y orientada. Como causa y consecuencia de ello, las agendas de las organizaciones internacionales de educación, las leyes educativas y los mal llamados sistemas 'educativos' construyen 'educaciones' sobre errores, insuficiencias y excesos. Los currícula no son ajenos a esta ineficacia pedagógica, global y profunda a la vez.

\section{1. ¿LOS CURRÍCULA SIRVEN PARA EDUCAR?}

Un currícula que no se ocupe de lo que más importa al ser humano, se desacredita como educativo. No lo será, por ejemplo, si no se orienta:

- a la formación de personas completas, y no sólo competentes (Savater, 1991);

- a amar, más allá del egoísmo;

- a la compasión, más allá de las crecientes insensibilidad, psicopatía y odios;

- a saberse sobre todo ignorante, y no sólo sabedor;

- a entender en qué consiste la discapacidad intelectual general asociada a la conciencia ordinaria, y no sólo que hay personas con discapacidad intelectual;

- a reconocer y salir de las propias cavernas (Platón), a superar dependencias de toda clase de ubres, y no sólo a existir;

- a la atención a la identidad, la semejanza y la unicidad, y no sólo a la diversidad;

- a la humanidad y la unidad del ser humano, $y$ no al nacionalismo, etnocentrismo y clasismo, en general; 
- a evolucionar, y no sólo a progresar;

- la finitud y la muerte, y no sólo a la 'educación para la vida';

- a interiorizarse, y no sólo a interesarse por el mundo;

- a meditar, y no sólo a pensar, analizar, sintetizar, deducir, preguntarse y responderse;

- a autoconocerse, y no sólo a conocer la existencia;

- a la conciencia, y no sólo a la adquisición de conocimientos mediante aprendizajes significativos y relevantes, etc.

En definitiva, si no ayuda a despertar, más allá de la ignorancia, la inconsciencia y la inmadurez generalizadas en que normalmente vivimos.

Si un currículo no atiende estos temas o retos formativos radicales, no contribuirá a la educación, aunque lo tenga por escrito o aunque diga que lo hace. Como la realidad es que las educaciones y los currícula se mueven exclusivamente a demanda de la sociedad, no son cabalmente formativos, porque no toda necesidad educativa se demanda.

El pozo (reto pedagógico) no es demasiado profundo. Lo que ocurre es que la cuerda (conciencia, ciencia, voluntad) es demasiado corta. Esta ineficacia no es propia de hoy, es un factor perenne. El ser humano tiende a no entrar por el atrio, a pesar de no tener sus puertas cerradas.

\section{2. ¿LOS CURRÍCULA PUEDEN SER PLENAMENTE EDUCATIVOS?}

Se dice que los currícula actuales son instrumentos abiertos y flexibles -aunque no lo sean-. Se reconoce que son incompletos, mirando a su sociedad y a su futuro profesional, tecnológico, económico... Se escucha menos que planifican aberraciones pedagógicas o que se desarrollan sobre el error. Para concluir con esto, quizá sea preciso el paso del tiempo y/o la evolución en complejidad-conciencia que tiende a traer.

Cuando el edificio está mal pensado, planificado y construido, cuando es peligroso por estar en un terreno inestable, no cumplirá su función, con independencia de que en alguna estancia se pueda estar pintando un bonito cuadro. Si sus deficiencias no se detectan, su evaluación será negligente. Por lo que respecta a los currícula, los perjuicios son tan antiguos, están tan extendidos y tan ocultos bajo los aciertos, que apenas se perciben.

Ahora bien, ¿pueden los currícula educar plenamente, y no sólo medio educar? Respondemos desde dos perspectivas:

- Desde una visión externa, hay que salir de los sistemas educativos. Si sus sistemas sociales son egocéntricos (inmaduros); si están condicionados conforme a su parcialidad/sesgo; si los intereses dominantes no son formativos, sino extraeducativos, etc., ¿cómo podrían no estarlo? Los currícula adoctrinarán mientras educan, y no propiciarán una educación plena.

- Desde una interna, se observan varios errores curriculares radicales, que impiden la educación plena:

a) Error conceptual fundamental: Se refiere al significado de la educación, cuya superficialidad general y radicalidad potencial no parecen percibirse con claridad por la Pedagogía, ni traducirse de un modo sencillo. La educación no sólo es externa (formulada, demandada, investigada, consensuada, existencial); es también interna (ignorada, no demandada, intuida, extraordinaria, esencial). Por tanto, o 
incluye el ego humano y la meditación, el conocimiento y la conciencia humana, el aprendizaje y el descondicionamiento, o no es de educación de lo que hablamos. Desde Sócrates hasta el siglo XXI no ha habido más que pseudoeducación en los sistemas de educación formal, hoy globalizados.

b) Error teleológico fundamental: Se refiere a la orientación sesgada de sus fines educativos, derivados de un concepto de educación radicalmente carente y erróneo. Los currícula responden a fines de la educación incompletos. Los fines de la educación consensuados no abarcan toda la educación. Sólo se refieren a lo que se ha llamado 'educación', que dista una corta infinidad con el fenómeno.

c) Error teleológico descarado: Se refiere a que, con la referencia puesta en sus sistemas sociales, nacionales $\mathrm{o}$ internacionales egocéntricos, los currícula mezclan educación y adoctrinamiento, llamando al resultado 'educación'. Esto es lo que define el 'currículo descarado'. Lo opuesto a educar es adoctrinar. Todos los sistemas educativos adoctrinan mientras educan, y lo hacen inconscientemente, por falta de conciencia pedagógica radical. Es una práctica general, del todo extendida y pertinente en la sociedad del egocentrismo. ¿Podrían estar todos equivocados? La respuesta es afirmativa. Los adoctrinamientos condicionan, encierran en cavernas $\mathrm{y}$, al hacerlo, las refuerzan constructivamente programando mentes, colonizando la razón sin decencia, porque aparentemente favorece la rentabilidad de su suprasistema social de referencia. Finalmente, despersonalizan al ciudadano, impeliendo pensamientos previsibles. Así, la persona se aleja de la autarquía sostenida en una razón propia (Kant) y se entrega al vasto campo de la estupidez humana. No es 'currículo oculto' porque se planifica; no es 'currículo nulo', porque se enseña; es "currículo descarado" (Herrán y González, 2002), porque se diseña, se desarrolla, se evalúa y se denomina 'educación', sin serlo en absoluto. Esta clase de currículo real es fundamental, para poder anhelar intenciones educativas conscientes del hielo de las alas. Con las alas lastradas podremos imaginar que volamos bien, pero ese espejismo seguirá formando parte de la ficción, de la inautenticidad, de la estafa consentida.

d) Error psi: Alude al excesivo protagonismo de la Psicología para cuestiones pedagógicas. El objeto de estudio de la Psicología, como ciencia de la salud, no es la educación. La ciencia cuyo objeto de estudio es la educación, la enseñanza, la formación y todo lo relacionado con ellos se llama 'Pedagogía'. Con una Pedagogía fuerte, la sociedad entera gana. Es un error de cultura general-común en periodistas, en políticos, en universitarios...- confundir Medicina y Farmacia, Arquitectura e Ingenierías, Geografía y Geología. También lo es confundir Pedagogía con Psicología o con disciplinas afines. La sobredosis psicológica no es buena para la educación.

e) Error molecular: Se refiere a la creencia, basada en la ambliopía de la Pedagogía y la Psicología, en que los sillares de los currícula son sólo los aprendizajes. Es decir, que el vector 'aprendizaje-conocimiento' es el atrio de la educación. Esta ofuscación, acorde con los errores anteriores, se traduce en una desorientación teleológica de la enseñanza y de la educación, a nivel molecular. Aprender es adquirir conocimientos; pero la educación, que por ende está basada en la conciencia, requiere perder, descondicionarse, soltar, no-saber, detener el pensamiento, la razón, vaciarse. 
f) Error personal: Se refiere a la orientación teleológica formativa del currículo, desde y hacia lo demandado, lo existencial, lo profesional y lo que conviene al desarrollo/ progreso de sus sistemas sociales. No toda necesidad educativa se demanda. Se precisa la armonía de las dos tendencias. Reducir la educación a demanda disciplinar y transversal y centrar esa necesidad en lo social y lo profesional es abandonar la formación personal, de raíz. El equivalente en Medicina sería paliar síntomas y renunciar a curar, algo absurdo que la propia sociedad no permitiría, porque sí sería consciente de ello.

g) Error descompensatorio: Se define por la dinámica del par de fuerzas: 'hipertrofia del enfoque competencial' y 'atrofia de enfoque de conciencia'. Se apoya en la tesis siguiente: centrar la educación en competencias es una contradicción formativa (Herrán, 2005) o una claudicación pedagógica.

h) Error dimensional: Se refiere a la insuficiente bidimensión curricular actual, compuesta por el eje disciplinar-competencias específicas, y el transversal-competencias comunes. Su interacción define un sistema plano, sin profundidad y que, en conjunto, conformaría el 'currículo demandado' socialmente. El eje faltante es el radical, generado por complejidad de conciencia, y definiría una tercera dimensión, compuesta por 'temas radicales' concretos. Sus características diferenciales serían: (1) No forman parte de las agendas de las organizaciones internacionales de educación, ni de los sistemas educativos, ni de la Pedagogía normal; (2) Son universales, independientes de contextos o comunes a todos ellos; (3) Son perennes o independientes de épocas o comunes a todas ellas; (4) En el momento actual, no se demandan socialmente; (5) No son ajenos a los docentes más conscientes, con independencia de su nivel de enseñanza;

(6) Son fundamentales para la educación;

(7) Apenas están desarrollados y (8) Son raíces de un árbol que es la educación de la conciencia. Los temas radicales son de dos tipos: deseables e indeseables. (1) Entre los deseables podrían expresarse: la conciencia (conceptos, estados, vías, etc.), la muerte y la finitud, el amor, la ternura y la compasión, la humanidad, la universalidad y la evolución humana (interior o educativa y exterior), el lenguaje universal, la madurez personal y social, la duda y la humildad, la voluntad, las enseñanzas de maestros/as despiertos, el autoconocimiento (esencial), la educación prenatal, la meditación, etc. (2) Entre los indeseables destacarían la ignorancia ignorante de sí misma, el egocentrismo y el condicionamiento, la necedad o estulticia, la inmadurez generalizada, la inconsciencia, la predisposición y el prejuicio, el fanatismo y el odio, el clasismo, ampliamente entendido, el sufrimiento, la insensibilidad y psicopatía crecientes, la barbarie, la discapacidad intelectual de quienes piensan que son otros los que la tienen, algunos de cuyos factores son la miopía, la dualidad, la parcialidad, el sesgo, etc.), etc.

i) Error perceptivo general: Se refiere al acostumbramiento de la vista a todos los errores anteriores. Un corolario es que cuesta reconocerlos. La principal dificultad para la toma de conciencia no depende tanto del fenómeno, cuanto del ego observador (científico, docente, padre-madre, estudiante, político, medios...), que, por su quietismo, 'no quiere querer' admitir que, quizá, pueda ver poco o mal. Esta dificultad no existe en quienes su razón gravita sobre la conciencia, que incluye la apertura, la duda y la humildad científica o profesional. Por tanto, más que de resolver problemas, se trata de definir problemas, admitirlos y de comprender su alcance educativo. Una 
vez pasada esta barrera de conciencia o de visión, podrían aparecer las pegas, las objeciones o los 'sí, pero...'. Una de las más destacadas suele venir de la imposibilidad de incorporar la dimensión radical, con su diversidad de ámbitos deseables e indeseables, al currículo. Las respuestas son dos: la primera es que, sin ellos bien interiorizados y planificados, la educación no puede darse, se queda antes de la mitad de camino. La segunda es que su desarrollo no ocupa lugar en la enseñanza: su cultivo se atiende con un enfoque radical, esto es, en uno mismo, porque cada conciencia, personal o social, es el centro donde posar el compás para trazar la circunferencia de la práctica y de los resultados educativos y sociales anhelados. Una vez atendido el interior, la enseñanza a otros (por ejemplo, alumnos), es espontánea, automática, y se comunica desde el ser y el no ser del o de la docente.

La dinámica pedagógica entre estos errores y los aciertos curriculares produce planificaciones curriculares incompletas, que subyacen y nutren los resultados sociales y personales caracterizados por el desequilibrio, el desarrollo exterior, la inconsciencia global y la escasa ambición evolutiva.

¿Están los currícula radicalmente equivocados? Sí, si lo que pretendiesen fuera educación o formación plenas. No, si su intención fuese un aglomerado compuesto de preparación, capacitación, exteriorización y adoctrinamiento.

\section{ALGUNAS ALTERNATIVAS RADICALES PARA LOS CURRÍCULA}

Las siguientes son alternativas de cambio radical de los currícula para las próximas décadas, que debería liderar y realizar, en buena lógica, la Pedagogía:

\section{INCLUSIÓN CURRICULAR DE LA CONCIENCIA}

Desde una perspectiva pedagógica radical e inclusiva, la educación consiste en perder ego y ganar conciencia, para vivir lo más despiertos posible. El binomio ego-conciencia es la clave de la formación, de la educación. La complejidad de conciencia es el vector resultante que describe, a la vez, la evolución de la educación y la conciencia de humanidad. Este hecho es simultáneamente externo e interno, superficial y profundo, personal y social.

La conciencia así conceptualizada no se incluye en la educación o el currículo. El despertar de la conciencia no es un fin de la educación. A lo sumo, una vez aplicada y expresada, pierde su sentido radical y gana el sustantivo. Entonces, se habla de conciencia medioambiental o de injusticia, de conciencia inclusiva o de violencia de género, etc., que absorben la atención social y pedagógica. Pero la educación necesita tanto conciencia aplicada como esencial. La conciencia radical se refiere a lo causal, al origen de la educación, comenzando por el observador, el dirigente, el educador...

La exclusión educativa y social de la conciencia está en la base de ausencias curriculares muy significativas. Cuando hay acción sin conciencia, no hay educación. Una educación y un currículo inconscientes hacen cosas bien y mal a la vez. La inconsciencia no está lejos de la estulticia. Donde hay conciencia, la ética es automática. Se necesitan razón y lucidez relativas, tanto sobre lo que construimos como sobre lo que dejamos de hacer.

Como los mayores problemas del ser humano son globales y están causados, en última instancia, por el ego y la conciencia ordinaria -que es débil, fragmentaria, parcial, dual y está condicionada-, las alternativas han de ser globales y polivalentes. 


\section{ALTERNATIVAS Y PROPUESTAS}

a) Propuesta epistemológica general: Fundamentar los currícula en: (1) Una revisión radical del concepto de educación, completándolo con el binomio egoconciencia. Sin lo que el ego y la conciencia humanos implican, la educación no es posible. (2) Una noción expandida de cultura que identifique a cada persona sobre todo con la humanidad. (3) La evolución humana, interior y exterior, más allá de la idea de progreso o desarrollo.

b) Propuesta epistemológica históricogenética: Revisar radicalmente la Pedagogía y complementar la tradición socrática de la educación y los currícula occidentales -con base en la adquisición de conocimientos, el saber, la palabra, la respuesta, la pregunta, la acción, etc.-, con las del Oriente clásico y las tradiciones espirituales sin dios/dioses -con base en el desprendimiento, el silencio, el no hacer (wu wei), la meditación y el no saber-. O sea, completar pedagógicamente -no filosóficamente- la herencia de Sócrates con las enseñanzas de los Upanishads, Lao Tse, de Zhuang zi, de Lie zi, del Buda Sakiamuni, del yoga, del chan, del zen, de las culturas originarias, etc.

c) Propuesta epistemológica profiláctica: En general, con los currícula pasa como con el Ganges: siendo apreciado por la población, no se deja de intoxicar con residuos industriales y humanos. Así como para este río lo que más conviene es su desintoxicación, para la educación, de la que depende el futuro de la humanidad, se propone eliminar de las leyes educativas y de los currícula los adoctrinamientos de toda clase (religiosos, ideológicos, nacionalistas, internacionalistas, sexistas, racistas, clasistas, cientificistas, etc.), que nada tienen que ver con ella. Primero, diferenciando entre condicionamiento $y$ educación y transfiriendo a la sociedad en qué difieren. Después, erradicando consensuada y progresivamente los añadidos egocéntricos a la educación (ismos), con el aval del discurso de los organismos internacionales de educación y de los medios.

d) Pasar, decididamente, de una educación formal y un currículo demandado o bidimensional, a un currículo tridimensional o $3 D$, a la vez disciplinar, transversal y radical. El paso de currículo convencional o demandado (2 D: disciplinar y transversal) a currículo $3 \mathrm{D}$ se daría por la inclusión natural en la planificación educativa formal del 'currículo radical', esto es, el compuesto por los temas radicales, tanto indeseables como deseables, por un avance en la complejidad de conciencia, con las cauciones necesarias ya expresadas sobre formación radical.

\section{A MODO DE CONCLUSIÓN}

Se propone un 'proceso de radicación pedagógica' general, tanto de la ciencia de la educación por antonomasia -la Pedagogíacomo de su campo de investigación y profesional -la educación-. Es decir, de inclusión educativa y curricular de la dimensión radical de la educación (Herrán, 2014), considerado desde la formación y la conciencia de cada observador o protagonista personal o social, en la medida en que puede considerarse un sistema educativo potencial.

Se propone centrarse, inicialmente, en las agendas de las organizaciones internacionales de educación, en la formación de investigadores y profesionales de la educación y la enseñanza, con especial confianza en los pedagogos. Será necesario desprejuzgar -en su caso- y prestigiar a estos profesionales, por ser los mayores expertos en educación, enseñanza y formación de que se dispone. Sus siguientes sujetos preferentes serían complementarios 
a los propios de la educación normal o la Pedagogía alopática; a saber: países con gran desarrollo y escasa evolución interna, dirigentes, políticos, poderes fácticos (medios de comunicación, banca, iglesia...), familias y educadores naturales, equipos directivos de centros, educadores profesionales... y, final o simultáneamente, los alumnos o los hijos, que no son ni deben ser el único centro de la educación (Herrán, 2017).

Podría coadyuvar a un posible verdadero cambio de paradigma educativo, esto es, de mirada, de teoría, de nuevos/ancestrales temas, de fundamentación, de orientación, de formación, de actuación, de evaluación, de innovación, de investigación, de transferencia, de prospectiva educativa, etc. Necesitaría el apoyo y el concurso de todos los sistemas sociales, en la medida en que puedan comprender que pueden ser, en sentido estricto, sistemas educativos (Herrán, 1993).

En la educación formal, se tratará de pasar de un currículo basado en el aprendizaje a un currículo basado en la conciencia, que lo englobe, donde se enseñe a ver, a adquirir, a perder-descondicionarse-desprenderse, a meditar-despertar y a evolucionar desde uno mismo. No se tratará de una educación para la vida, sino de una educación para cambiar la vida radicalmente y para una vida más consciente. No habría que perder de vista las fases (no lineales) de la verdadera educación (Herrán, 2014), hoy completamente ignorada, y que sintetizamos a continuación: (1) Fase de condicionamiento (actual, conciencia ordinaria); (2) Fase de descondicionamiento (actual, conciencia extraordinaria); (3) Fase de recondicionamiento (actual, conciencia extraordinaria); (4) Fase de autoconocimiento (casi inédita); (5) Fase de vacío (casi inédita).
- Herrán Gascón, A. de la (2019). Por qué los currícula no pueden educar. Una mirada desde la Pedagogía radical e inclusiva. Cuadernos de Pedagogía (502), 75-80.

\section{REFERENCIAS BIBLIOGRÁFICAS}

- Herrán, A. de la (1993). La educación del siglo XXI. Cambio y evolución humana. Madrid: Ciencia 3.

- Herrán, A. de la (2005). Formación y transversalidad universitarias. Tendencias pedagógicas (10), 223-256. Recuperado de https://revistas.uam.es/ tendenciaspedagogicas/article/view/1862

- Herrán, A. de la (2012). Currículo y pedagogías renovadoras en la edad antigua. Revista iberoamericana sobre calidad, eficacia y cambio en educación, 10(4), 286334. Recuperado de http://www.rinace.net/ reice/numeros/arts/vol10num4/art17.pdf

- Herrán, A. de la (2014). Enfoque radical e inclusivo de la formación. REICE. Revista iberoamericana sobre calidad, eficacia y cambio en educación, 12(2), 163-264. Recuperado de: http://www.rinace.net/reice/ numeros/arts/vol12num2/art8.pdf

- Herrán, A. de la (2017). ¿Y si nuestra educación estuviese radicalmente equivocada? En A. Medina, A. de la Herrán y M. C. Domínguez (Coords.), Nuevas perspectivas en la formación de profesores. Madrid: UNED. Recuperado de http://radicaleinclusiva.com/wp-content/ uploads/2018/12/educacionequivocada.pdf 
- Herrán, A. de la (2018). Fundamentos para una Pedagogía del saber y del no saber. São Paulo: Hipótese. Recuperado de https:// drive.google.com/file/d/1dprFjWeqkKkOMSAxCCZpe UqU-64UnQ/view

- Herrán, A. de la y González, I. (2002). El ego docente, punto ciego de la enseñanza, el desarrollo profesional y la formación del profesorado. Madrid: Universitas.

- Herrán, A. de la, Valle, J. M. y Villena, J. L. (2019). ¿Qué estamos haciendo mal en la educación? Reflexiones pedagógicas para la investigación, la enseñanza y la formación. Barcelona: Octaedro.

- Savater, F. (1991). El valor de educar. Madrid: Ariel. 